\title{
Density of Skew Brownian motion and its functionals with application in finance
}

\author{
Alexander Gairat*and Vadim Shcherbakov ${ }^{\dagger}$
}

\begin{abstract}
We derive the joint density of a Skew Brownian motion, its last visit to the origin, its local and occupation times. The result allows to obtain explicit analytical formulas for pricing European options under both a two valued local volatility model and a displaced diffusion model with constrained volatility.
\end{abstract}

Key words: Skew Brownian motion, local volatility model, displaced diffusion, local time, occupation time, simple random walk, option pricing

\section{Introduction}

A Skew Brownian motion (SBM) with parameter $p$ is a Markov process that evolves as a standard Brownian motion reflected at the origin so that the next excursion is chosen to be positive with probability $p$. SBM was introduced in [18] and has been studied extensively in probability since then. The process naturally appears in diverse applications, e.g. [3] and [20], and, in particular, in finance applications, e.g. [5], 6], [7] and [24]. In this paper we derive the joint distribution of SBM and some of its functionals and apply this distribution to derivative pricing under both a local volatility model with discontinuity and a displaced diffusion model with constrained volatility.

Let $(\Omega, \mathcal{F}, \mathrm{P})$ be a probability space and let $\left\{W_{t}, \mathcal{F}_{t}, t \geq 0\right\}$ be a standard Brownian motion (BM) with its natural filtration. As usual, denote by $\mathbb{R}$ and $\mathbb{R}_{+}$sets of all real and all nonnegative real numbers respectively. A local volatility model (LVM) for the underlying price $S_{t}$ is given by the following equation

$$
d S_{t}=\mu(t) S_{t} d t+\sigma\left(t, S_{t}\right) S_{t} d W_{t}
$$

where $\mu(t) \in \mathbb{R}$ and $\sigma\left(t, S_{t}\right) \in \mathbb{R}_{+}$. LVM is a natural extension of the famous Black-Scholes model. The latter is a particular case of (10) where both drift $\mu$ and volatility $\sigma$ are constant. LVM is actively used in practice because it can be easily calibrated to the market. Furthermore, by Gyongy's lemma ([13]) a wider class of stochastic volatility models can be reduced to LVM.

\footnotetext{
*Gazprombank, Moscow. Email address: Alexander.Gairat@gazprombank.ru

${ }^{\dagger}$ Department of Mathematics, Royal Holloway, University of London. Email address: vadim.shcherbakov@rhul.ac.uk
} 
A number of approximations to LVM have been developed for both calibration purposes and qualitative analysis ([12]). We apply our probabilistic results primarily to a particular case of LVM that can be used as a benchmark model for analyzing quality of such approximations. Namely, we consider a driftless LVM with a two-valued volatility (two-valued LVM)

$$
\sigma(t, S)=\sigma_{1} 1_{\left\{S \geq S^{*}\right\}}+\sigma_{2} 1_{\left\{S<S^{*}\right\}}
$$

where $\sigma_{i}>0, i=1,2, S^{*}>0$, and $1_{A}$ is used to denote the indicator function of set $A$. Without loss of generality we assume that $S^{*}=1$ in what follows.

In Section 3.1 we show that if $S_{t}$ follows the two-valued LVM then rescaled process $X_{t}=$ $\log \left(S_{t}\right) / \sigma\left(S_{t}\right)$ is a solution of a stochastic differential equation (SDE) of the following type

$$
X_{t}=X_{0}+\int_{0}^{t} m\left(X_{s}\right) d s+(2 p-1) L_{t}^{(0)}(X)+W_{t}
$$

where $L_{t}^{(0)}(X)$ is the local time of process $X_{t}$ at zero, $p \in(0,1)$,

$$
m(x)=m_{1} 1_{\{x \geq 0\}}+m_{2} 1_{\{x<0\}}, m_{1}, m_{2} \in \mathbb{R},
$$

and both $p$ and pair $\left(m_{1}, m_{2}\right)$ are uniquely determined by $\sigma_{1}$ and $\sigma_{2}$ (Lemma 1). Notice that SDE (13) belongs to the following class of SDE with local time

$$
d X_{t}=b\left(X_{t}\right) d t+\sigma\left(X_{t}\right) d W_{t}+\int_{\mathbb{R}} \nu(d x) d L_{t}^{(x)}(X),
$$

where $\nu$ is a finite signed measure with atoms at the points, where both $b$ and $\sigma$ can be discontinuous, and $L_{t}^{(x)}(X)$ is the local time of process $X$ at $x$. It is known that SDE (5) has a unique strong solution under certain general conditions which are satisfied in the case of equation (3) (e.g. [19], [20] and references therein). In particular, if $m \equiv 0$ then a unique strong solution of equation (3) is a SBM with parameter $p$ which we are going to denote by $W_{t}^{(p)}$ from now on. If $m_{1}=m_{2}=m$, then equation (3) takes the following form

$$
X_{t}=X_{0}+m t+(2 p-1) L_{t}^{(0)}+W_{t}
$$

A diffusion process defined by equation (6) appears, for instance, in a study of dispersion across an interface in [3] and is named there as a SBM with parameter $p$ and drift $m$. By analogy, we refer to the solution of equation (3) as a SBM with a two-valued drift. A SBM with two-valued drift (44) is reflected at the origin in the same way as a driftless $W_{t}^{(p)}$ and evolves as a BM with drift $m_{1}$ when it is above zero and, respectively, with drift $m_{2}$ when it is below zero.

In general, option prices under LVM are calculated by solving numerically the corresponding partial differential equations, though some semi-analytical results are also known. For example, semi-analytical results have been obtained in [6] for LVM where $\sigma(t, S)=\sigma(S)$ is continuous at all but one point. Another example is provided by [21], where semi-analytical results have been obtained for LVM with a so-called tiled local volatility. 
We derive explicit formulas for the joint density of $W_{t}^{(p)}$, its last visit to the origin, local and occupation times in both the driftless and the two-valued drift cases. The joint density is then applied to option pricing under both LVM with volatility (2) and a displaced diffusion model with constrained volatility. The second model is defined in Section 6 and is of a particular interest in practical applications. It should be noted that both models belong to the more general class of LVM considered in [6]. It turns out that European option prices in both cases can be expressed analytically in terms of both a standard univariate normal distribution and a bivariate normal distribution.

Joint distributions of SBM and its functionals are of interest on their own right. For example, the joint density of SBM with a constant drift, its local and occupation times was obtained in [3]. The result of [3] generalizes the classic result of [16], where the same trivariate density was obtained for the standard BM. In [3] the technique of [18] was modified to obtain a FeynmanKac formula for SBM and this allowed them to adopt the method of [16]. In turn, the method of [16] is based on the computation of the Laplace transform of the joint density. In contrast, we use a discrete approximation of SBM by a random walk and a key step of our approach consists in combining an intuitively clear path decomposition for the discrete process with some well known properties of the symmetric simple random walk. This allows us to derive analytically tractable expressions for the joint density of discrete analogues of quantities of interest and to compute the limit density.

A discrete approximation is a well known method for obtaining joint distribution of both BM and SBM and their functionals (e.g. [22] or [25]). We were inspired by the use of this method in [4] for computation of the joint distribution of the standard BM, its both the occupation time and the last visit to the origin.

The paper is organized as follows. We formulate the results on the joint distribution of SBM and its functionals in Section 2. In Section 3 we describe the relationship between LVM with the two-valued volatility and SBM with the two-valued drift. Theorem 3 in Section 3.2 is an example of an option pricing theorem under the two-valued LVM. Proofs are given in Section 4 . In Section 5 we also derive a simple closed form approximation for option prices based on the Black-Scholes formula. Effectiveness of the approximate result is tested in comparison with the exact result provide by Theorem 3 and another approximation obtained in [21]. Finally, we discuss in Section 6 how our results can be applied to derivative pricing under the displaced diffusion model with constrained volatility. 


\section{Density of Skew Brownian motion, its last visit to the origin, occupation and local times}

Given a continuous semimartingale $X_{t}, t \in[0, T]$, define the following quantities

$$
\begin{aligned}
\tau_{0} & =\min \left\{t: X_{t}=0\right\} \\
\tau & =\max \left\{t \in(0, T]: X_{t}=0\right\}, \\
V & =\int_{\tau_{0}}^{\tau} 1_{\left\{X_{t} \geq 0\right\}} d t .
\end{aligned}
$$

Also, let $L_{t}^{(x)}(X)$ be the symmetric local time of $X_{t}$ at point $x$. In particular, if $X_{t}=W_{t}^{(p)}$, or $X_{t}$ is the solution of equation (3) then

$$
L_{t}^{(x)}(X)=\lim _{\varepsilon \rightarrow 0} \frac{1}{2 \varepsilon} \int_{0}^{t} 1_{\left\{x-\varepsilon \leq X_{u} \leq x+\varepsilon\right\}} d u .
$$

In what follows we consider only the symmetric local time (the local time).

Our principal result about joint density of SBM and its functionals is the following theorem.

Theorem 1 Let $\left(\tau, V, L_{T}^{(0)}(X)\right)$ be the quantities defined by equations (8), (9) and (10) with $X_{t}=W_{t}^{(p)}$. Given $X_{0}=0$ the joint density of $\left(\tau, V, X_{T}, L_{T}^{(0)}(X)\right)$ is

$$
\psi_{p, T}(t, v, x, l)=2 a(x) h(v, l p) h(t-v, l q) h(T-t, x), 0 \leq v \leq t \leq T, l \geq 0,
$$

where $q=1-p$,

$$
a(x)= \begin{cases}p, & \text { if } x \geq 0 \\ q, & \text { if } x<0\end{cases}
$$

and

$$
h(s, y)=\frac{|y|}{\sqrt{2 \pi s^{3}}} e^{-\frac{y^{2}}{2 s}}, y \in \mathbb{R}, s \in \mathbb{R}_{+},
$$

is the probability density function of the first passage time to zero of the standard BM starting at $y$.

Theorem 2 Let $\left(\tau, V, L_{T}^{(0)}(X)\right)$ be the quantities defined by equations (8), (9) and (10) for a solution $X_{t}$ of equation (3). Given $X_{0}=0$ the joint density of $\left(\tau, V, X_{T}, L_{T}^{(0)}(X)\right)$ is given by the following function

$$
\phi_{T}(t, v, x, l)=\psi_{p, T}(t, v, x, l) e^{-\frac{m_{1}^{2} v+m_{2}^{2}(T-v)}{2}-l\left(m_{1} p-q m_{2}\right)+x m(x)}, 0 \leq v \leq t \leq T, l \geq 0,
$$

where $\psi_{p, T}(t, v, z, l)$ is defined by equation (11). 
Let us briefly comment on relationship of Theorems 1 and 2 with some known results. First we rewrite the joint density (13) in terms of the total occupation time. Given $T>0$ define

$$
U=\int_{0}^{T} 1_{\left\{X_{t} \geq 0\right\}} d t
$$

the total occupation time of the non-negative half-line during time period $[0, T]$ and notice that if $X_{0}=0$ then

$$
U= \begin{cases}V+T-\tau, & \text { if } X_{T} \geq 0, \\ V, & \text { if } X_{T}<0 .\end{cases}
$$

If $X$ is SBM with parameter $p$ and drift $m(x)$ then Theorem 11 and equation (14) yield that the joint density of $\left(\tau, U, X_{T}, L_{T}^{(0)}(X)\right)$ is given by the following equation

$$
\varphi_{T}(t, u, x, l)=\left\{\begin{array}{l}
2 p h(u+t-T, l p) h(T-u, l q) h(T-t, x) e^{-\frac{m_{1}^{2} u+m_{2}^{2}(T-u)}{2}+x m_{1}-l\left(p m_{1}-q m_{2}\right)}, \\
\text { if } x \geq 0, l>0, \text { and } t \leq T, T-t \leq u \leq T, \\
2 q h(u, l p) h(t-u, l q) h(T-t, x) e^{-\frac{m_{1}^{2} u+m_{2}^{2}(T-u)}{2}+x m_{2}-l\left(p m_{1}-q m_{2}\right)}, \\
\text { if } x<0, l>0, \text { and } 0 \leq u \leq t \leq T .
\end{array}\right.
$$

If $m_{1}=m_{2}=m=$ const then we obtain the density of the quartet in the case of the constant drift

$$
\varphi_{T, m}(t, u, x, l)=\left\{\begin{array}{l}
2 p h(u+t-T, l p) h(T-u, l q) h(T-t, x) e^{-\frac{m^{2} T}{2}+x m-l m(p-q)}, \\
\text { if } x \geq 0, l>0, \text { and } t \leq T, T-t \leq u \leq T \\
2 q h(u, l p) h(t-u, l q) h(T-t, x) e^{-\frac{m^{2} T}{2}+x m-l m(p-q)} \\
\text { if } x<0, l>0, \text { and } 0 \leq u \leq t \leq T
\end{array}\right.
$$

Further, setting $m=0$ in the preceding display and integrating out variable $t$ we get the joint density of SBM with parameter $p$, its (total) occupation and local time (Theorem 1.2 in [3])

$$
\begin{aligned}
\rho(u, z, b) & = \begin{cases}\int_{0}^{T} 2 p h(u+t-T, l p) h(T-u, l q) h(T-t, x) d t, & x \geq 0, \\
\int_{u}^{T} 2 q h(u, l p) h(T-u, l q) h(T-t, x) d t, & x<0,\end{cases} \\
& = \begin{cases}2 p h(T-u, b q) h(u, l p+x), & x \geq 0, \\
2 q h(u, l p) h(T-u, l q-x), & x<0 .\end{cases}
\end{aligned}
$$

In a particular case $p=1 / 2$ density (16) is the trivariate density obtained in [16] for the standard BM. It should be noticed that the local time in [16] equals to a half of the local time defined by (10). 


\section{Application in finance}

\subsection{Relationship between LVM with discontinuity and SBM}

Fix $\sigma_{1}>0$ and $\sigma_{2}>0$ and consider the following LVM

$$
d S_{t}=\sigma\left(S_{t}\right) S_{t} d W_{t}
$$

where

$$
\sigma(S)=\sigma_{1} 1_{\{S \geq 1\}}+\sigma_{2} 1_{\{S<1\}} .
$$

Lemma 1 below explains the relationship between SBM and LVM defined by (17). This lemma can be regarded as a particular case of Theorem 1 in [6]) (see also an argument on p.687 in [5]) and is based on application of the symmetric Tanaka-Meyer formula (e.g. see either formula (7.4) in [17, or Exercise 1.25, Chapter VI in [23], or formula (32) in [20]). We provide the proof here for the sake of completeness and for the reader's convenience.

Lemma 1 Let $S_{t}$ be a solution of equation (17). A stochastic process $X_{t}$ defined as follows

$$
X_{t}=\frac{\log \left(S_{t}\right)}{\sigma\left(S_{t}\right)}
$$

is a solution of the following SDE with the local time

$$
d X_{t}=\mu\left(X_{t}\right) d t+d W_{t}+(p-q) d L_{t}^{(0)}(X)
$$

where

$$
\mu(x)=-\frac{\sigma\left(e^{x}\right)}{2}= \begin{cases}\mu_{1}=-\sigma_{1} / 2, & x \geq 0 \\ \mu_{2}=-\sigma_{2} / 2, & x<0\end{cases}
$$

and

$$
p=\frac{\sigma_{2}}{\sigma_{1}+\sigma_{2}}, q=1-p=\frac{\sigma_{1}}{\sigma_{1}+\sigma_{2}} .
$$

In other words, $X_{t}$ is SBM with parameter $p=\frac{\sigma_{2}}{\sigma_{1}+\sigma_{2}}$ and discontinuous drift $\mu(x)$.

Proof of Lemma 1. First, define $Y_{t}=\log \left(S_{t}\right)$ and notice that by usual Ito's formula

$$
d Y_{t}=-\frac{\sigma^{2}\left(S_{t}\right)}{2} d t+\sigma\left(S_{t}\right) d W_{t}=-\frac{\sigma^{2}\left(e^{Y_{t}}\right)}{2} d t+\sigma\left(e^{Y_{t}}\right) d W_{t}
$$

In terms of process $Y_{t}$ we have that $X_{t}=f\left(Y_{t}\right)$, where $f(y)=\frac{y}{\sigma_{1}} 1_{\{y \geq 0\}}+\frac{y}{\sigma_{2}} 1_{\{y<0\}}$. It is easy to see that $f$ is a difference of two convex functions and, hence, $X_{t}=f\left(Y_{t}\right)$ is a semimartingale. Define $f^{\prime}(y)=\frac{1}{2}\left(f_{l}^{\prime}(y)+f_{r}^{\prime}(y)\right)$, where $f_{l}^{\prime}(y)$ and $f_{r}^{\prime}(y)$ are the left and the right derivative of $f$ respectively. It is easy to see that

$$
f^{\prime}(y)=\frac{1}{\sigma(y)} 1_{\{y \neq 0\}}+\frac{\sigma_{1}+\sigma_{2}}{2 \sigma_{1} \sigma_{2}} 1_{\{y=0\}} .
$$


Also, the second derivative of $f$ (in the distribution sense) is $f^{\prime \prime}(y)=\delta(y)\left(\frac{1}{\sigma_{1}}-\frac{1}{\sigma_{2}}\right)$, where $\delta(x)$ is the delta function. Applying the symmetric Tanaka-Meyer formula to semimartingale $f\left(Y_{t}\right)$ we get that

$$
\begin{aligned}
X_{t}=f\left(Y_{t}\right) & =f\left(Y_{0}\right)+\int_{0}^{t} f^{\prime}\left(Y_{u}\right) d Y_{u}+\int_{\mathbb{R}} f^{\prime \prime}(y) L_{t}^{(y)}(Y) d y \\
& =f\left(Y_{0}\right)+\int_{0}^{t}\left(\frac{1}{\sigma(y)} 1_{\{y \neq 0\}}+\frac{\sigma_{1}+\sigma_{2}}{2 \sigma_{1} \sigma_{2}} 1_{\{y=0\}}\right) d Y_{u}+\frac{1}{2}\left(\frac{1}{\sigma_{1}}-\frac{1}{\sigma_{2}}\right) L_{t}^{(0)}(Y) \\
& =X_{0}-\int_{0}^{t} \frac{\sigma\left(e^{X_{u}}\right)}{2} d u+W_{t}+\frac{1}{2}\left(\frac{1}{\sigma_{1}}-\frac{1}{\sigma_{2}}\right) L_{t}^{(0)}(Y)
\end{aligned}
$$

where $L_{t}^{(0)}(Y)$ is the local time of $Y_{t}$ at zero and where we also used that $\int_{0}^{t} 1_{\left\{Y_{u}=0\right\}} d Y_{u}=0$ and $\sigma\left(e^{Y_{t}}\right)=\sigma\left(e^{X_{t}}\right)$, in order to get equation (24) from equation (23).

It is left to express $L_{t}^{(0)}(Y)$ in terms of $L_{t}^{(0)}(X)$. Firstly, we apply symmetric Tanaka-Meyer formula to semimartingale $X_{t}$ with convex function $|x|$ and get that

$$
\left|X_{t}\right|=\left|X_{0}\right|+\int_{0}^{t} \operatorname{sgn}\left(X_{u}\right) d X_{u}+L_{t}^{(0)}(X)
$$

where $\operatorname{sgn}(x)=1$ if $x>0, \operatorname{sgn}(x)=-1$, if $x<0$, and $\operatorname{sgn}(0)=0$. Secondly, consider $\left|X_{t}\right|$ as a result of applying convex function $g(y)=|f(y)|=\frac{y}{\sigma_{1}} 1_{\{y \geq 0\}}-\frac{y}{\sigma_{2}} 1_{\{y<0\}}$ to semimartingale $Y_{t}$. Let $g^{\prime}$ be the arithmetic mean of the right and the left derivatives of $g$. It is easy to see that

$$
g^{\prime}(y)=\frac{1}{2}\left(g_{l}^{\prime}(y)+g_{r}^{\prime}(y)\right)=\operatorname{sgn}(y) \frac{1}{\sigma(y)}+\frac{1}{2}\left(\frac{1}{\sigma_{1}}-\frac{1}{\sigma_{2}}\right) 1_{\{y=0\}} .
$$

The second generalised derivative $g^{\prime \prime}$ of $g$ is $\left(\frac{1}{\sigma_{1}}+\frac{1}{\sigma_{2}}\right) \delta(y)$. Applying symmetric Tanaka-Meyer formula to $g\left(Y_{t}\right)$ we obtain that

$$
\left|X_{t}\right|=\left|f\left(Y_{t}\right)\right|=\left|X_{0}\right|+\int_{0}^{t} g^{\prime}\left(Y_{u}\right) d Y_{u}+\frac{1}{2}\left(\frac{1}{\sigma_{1}}+\frac{1}{\sigma_{2}}\right) L_{t}^{(0)}(Y) .
$$

Noticing that

$$
\begin{aligned}
\int_{0}^{t} \operatorname{sgn}\left(X_{u}\right) d X_{u} & =\int_{0}^{t} \operatorname{sgn}\left(Y_{u}\right) \frac{1}{\sigma\left(Y_{u}\right)}\left(-\frac{\sigma^{2}\left(e^{Y_{u}}\right)}{2} d u+\sigma\left(e^{Y_{u}}\right) d W_{u}\right) \\
& =\int_{0}^{t} g^{\prime}\left(Y_{u}\right) d Y_{u}-g^{\prime}(0) \int_{0}^{t} 1_{\left\{Y_{u}=0\right\}} d Y_{u}=\int_{0}^{t} g^{\prime}\left(Y_{u}\right) d Y_{u}
\end{aligned}
$$


and comparing right sides of equations (25) and (26) we obtain the following identity

$$
L_{t}^{(0)}(X)=\frac{1}{2}\left(\frac{1}{\sigma_{1}}+\frac{1}{\sigma_{2}}\right) L_{t}^{(0)}(Y)
$$

which yields that

$$
d X_{t}=-\frac{\sigma\left(e^{X_{t}}\right)}{2} d t+d W_{t}+\frac{\sigma_{2}-\sigma_{1}}{\sigma_{1}+\sigma_{2}} d L_{t}^{(0)}(X)=\mu\left(X_{t}\right) d t+d W_{t}+(p-q) d L_{t}^{(0)}(X)
$$

as claimed. Lemma 1 is proved.

Remark 1 Denote by $\mathbb{Q}_{T}$ the probability distribution of SBM with parameter $p$ and drift (44) on the time interval $[0, T]$ and by $\mathbb{P}_{T}$ the probability distribution of $W_{t}^{(p)}, t \in[0, T]$. By the Girsanov's theorem we have that

$$
\begin{aligned}
\frac{d \mathbb{Q}_{T}}{d \mathbb{P}_{T}}(X .) & =e^{\int_{X_{0}}^{X_{T}} m(u) d u-\frac{1}{2} \int_{0}^{T} m^{2}\left(X_{t}\right) d t-\left(p m_{1}-q m_{2}\right) L_{T}^{(0)}(X)} \\
& =e^{\int_{X_{0}}^{X_{T}} m(u) d u-\frac{1}{2} \int_{0}^{T} m^{2}\left(X_{t}\right) d t-\left(p m_{1}-q m_{2}\right) L_{T}^{(0)}(X)} \\
& =e^{\int_{x_{0}}^{x} m(u) d u-\frac{1}{2}\left(m_{1}^{2} w-m_{2}^{2}(T-w)\right)-\left(p m_{1}-q m_{2}\right) l}
\end{aligned}
$$

for any trajectory $X$. such that $X_{0}=x_{0}, X_{T}=x, \int_{0}^{T} 1_{\left\{X_{t} \geq 0\right\}} d t=w, L_{T}^{(0)}(X)=l$.

\subsection{Option pricing under the two-valued local volatility model}

In this section we show how the results of Section 2 can be applied to option pricing under the two-valued LVM. We do it by example in the case of a European call option. Recall first some terminology and facts of the option pricing theory. A European call option (call option) with strike price (strike) $K$ and expiration date $T$ is a derivative whose payoff is $\left(S_{T}-K\right)^{+}=\max \left(S_{T}-K, 0\right)$, where $S_{T}$ is the price of the underlying asset at expiration. A knock-in call option with barrier $H$ is a regular call option that comes into existence only when the underlying reaches the barrier. A knock-out call option with barrier $H$ is a regular call option that ceases to exist as soon as the underlying reaches the barrier.

Consider the two valued LVM defined by equations (17) and (18) (i.e. with discontinuity at $\left.S^{*}=1\right)$. Given value $S_{0}$ of the underlying at $t=0$, strike $K$ and expiry date $T$, denote by $\mathrm{C}=\mathrm{C}\left(S_{0}, K, T\right)$ and $\mathrm{C}_{\text {in }}=\mathrm{C}_{\mathrm{in}}\left(S_{0}, K, T\right)$ the price of a call option and the price of a knock-in call option with the barrier level of 1 respectively, where both prices are computed under the two-valued LVM. Also, given the same parameters denote by $\mathrm{C}_{\text {out }}=\mathrm{C}_{\text {out }}\left(S_{0}, K, T, \sigma_{1}, 1\right)$ the price of a knock-out call option with the barrier level of 1 computed under the log-normal model with volatility $\sigma_{1}$.

It is easy to see that if $K>1$ then

$$
\mathrm{C}= \begin{cases}\mathrm{C}_{\text {in }}+\mathrm{C}_{\text {out }}, & S_{0} \geq 1 \\ \mathrm{C}_{\text {in }}, & S_{0}<1\end{cases}
$$


Prices of barrier options under the log-normal model are known (e.g., see ch.22 in [15]). Therefore, if $K>1$ then it is only left to find $C_{\text {in }}$ under the two valued LVM in order to price a call option. A formula for the knock in call option price $C_{i n}$ is given by Theorem 3 below.

The price of a call option with strike $K<1$ and prices of put options can be obtained in a similar way. Notice that in the case of a call (put) option with strike $K<1(K>1)$ it seems technically more convenient to start with computing the price of a put (call) option with the same parameters and then to use the put-call parity equation.

Let us introduce some functions that will appear in Theorem 3 and its proof. Let

$$
\mathrm{n}(x)=\frac{e^{-\frac{x^{2}}{2}}}{\sqrt{2 \pi}}, x \in \mathbb{R}
$$

be the probability density function and

$$
\Phi(z)=\frac{1}{\sqrt{2 \pi}} \int_{-\infty}^{z} e^{-\frac{y^{2}}{2}} d y, z \in \mathbb{R}
$$

be the cumulative distribution function of the standard normal distribution. Let

$$
\mathcal{N}(x, y, \rho)=\int_{-\infty}^{x} \int_{-\infty}^{y} \frac{e^{\frac{1}{1-\rho^{2}}\left(-\frac{z_{1}^{2}}{2}-\rho z_{1} z_{2}+\frac{z_{2}^{2}}{2}\right)}}{2 \pi \sqrt{1-\rho^{2}}} d z_{1} d z_{2}, x, y \in \mathbb{R}
$$

be the joint cumulative distribution function of the bivariate normal distribution with zero means, unit variances and correlation $\rho$.

Also denote

$$
\phi(S)=\frac{\log (S)}{\sigma(S)}= \begin{cases}\log (S) / \sigma_{1}, & S \geq 1 \\ \log (S) / \sigma_{2}, & S<1\end{cases}
$$

and

$$
h(t, x, b)=\frac{x}{\sqrt{2 \pi t^{3}}} e^{-\frac{(x+b t)^{2}}{2 t}}, t \in \mathbb{R}_{+}, x, b \in \mathbb{R},
$$

Finally, for simplicity of notation, we assume in Theorem 3 that the risk-free interest rate is zero.

Theorem 3 Let $S_{t}$ be the random process that follows equation (17) with function (18). Given $K>0$ and $S_{0}>0$ denote $k=\phi(K)$ and $x_{0}=\phi\left(S_{0}\right)$. Let $\mathrm{C}_{\mathrm{in}}=\mathrm{C}_{\mathrm{in}}\left(S_{0}, K, T\right)$ be the price of a knock-in European call option with strike $K$ and expiration date $T$ given the initial price $S_{0}$.

1) If $S_{0} \geq 1, K>1$, then

$$
\mathrm{C}_{\text {in }}=p e^{-\frac{\sigma_{1} x_{0}}{2}}\left(F_{\text {call }}\left(\frac{\sigma_{1}}{2}, x_{0}\right)-e^{\sigma_{1} k} F_{\text {call }}\left(-\frac{\sigma_{1}}{2}, x_{0}\right)\right)
$$

where

$$
F_{\text {call }}\left(a, x_{0}\right)=\int_{0}^{T} F_{1}(T-t) F_{2}\left(a, t, x_{0}, 1\right) e^{-\frac{t \sigma_{1}^{2}}{8}} d t
$$


and where, in turn,

$$
\begin{aligned}
F_{1}(s) & =\frac{\sqrt{2}\left(\sigma_{1} e^{-\frac{\sigma_{2}^{2} s}{8}}-\sigma_{2} e^{-\frac{\sigma_{1}^{2} s}{8}}\right)+\sqrt{\pi s} \sigma_{1} \sigma_{2}\left(\Phi\left(\frac{\sqrt{s} \sigma_{2}}{2}\right)-\Phi\left(\frac{\sqrt{s} \sigma_{1}}{2}\right)\right)}{\sqrt{\pi s}\left(\sigma_{1}-\sigma_{2}\right)}, \\
F_{2}\left(a, t, x_{0}, \theta\right) & =\frac{1}{\sqrt{2 \pi} \sqrt{t}} e^{k a-\frac{\left(\left|x_{0}\right|+|k|\right)^{2}}{2 t}}+a e^{a\left|x_{0}\right|+\frac{t a^{2}}{2}}\left(1-\Phi\left(\theta \frac{\left|x_{0}\right|+|k|}{\sqrt{t}}-a \sqrt{t}\right)\right) .
\end{aligned}
$$

2) If $S_{0}<1, K>1$, then

$$
\begin{gathered}
\mathrm{C}_{\text {in }}=2 p e^{\frac{\sigma_{2} x_{0}}{2}}\left(G\left(-\frac{\sigma_{1}}{2}, x_{0}\right)-e^{k \sigma_{1}} G\left(\frac{\sigma_{1}}{2}, x_{0}\right)\right) \\
G\left(a, x_{0}\right)=\int_{0}^{T} e^{-\frac{\sigma_{1}^{2} v}{8}-\frac{\sigma_{2}^{2}(T-v)}{8}} e^{\frac{\sigma_{1}^{2}}{8}\left(u\left(\frac{p}{q}\right)^{2}+v\right)-\left|x_{0}\right| \frac{p a}{q}} G_{1}\left(\left(a, v,\left|x_{0}\right|,-\frac{p}{q} a\right) d v\right.
\end{gathered}
$$

where

$$
G_{1}(a, v, y, w)=\int_{k}^{\infty} \int_{0}^{\infty} h(v, l p+x, a) h(T-v, l q+y, w) d l d x .
$$

In turn, $G_{1}$ can be expressed in terms of the standard normal distribution (i.e. in terms of its pdf (28) and its cdf (29)) and a bivariate normal cdf (30) as follows

$$
\begin{aligned}
& G_{1}(a, y, v, w) q \sqrt{v(T-v)} \\
& \quad=\frac{\mathrm{n}(\gamma X+Y) \mathrm{n}(X)}{1+\gamma^{2}}-\frac{\gamma Y}{\left(1+\gamma^{2}\right)^{3 / 2}} \mathrm{n}\left(\frac{Y}{\sqrt{1+\gamma^{2}}}\right) \Phi\left(-\frac{\left(1+\gamma^{2}\right) X+\gamma Y}{\sqrt{1+\gamma^{2}}}\right) \\
& \quad-\frac{\alpha}{\sqrt{1+\gamma^{2}}} \mathrm{n}\left(\frac{Y}{\sqrt{1+\gamma^{2}}}\right) \Phi\left(-\frac{\left(1+\gamma^{2}\right) X+\gamma Y}{\sqrt{1+\gamma^{2}}}\right) \\
& \quad-\beta \mathrm{n}(X) \Phi(-\gamma X-Y)-\frac{\gamma}{\sqrt{2 \pi\left(1+\gamma^{2}\right)}} \mathrm{n}\left(\frac{Y}{\sqrt{1+\gamma^{2}}}\right) \Phi\left(-\frac{\left(1+\gamma^{2}\right) X+\gamma Y}{\sqrt{1+\gamma^{2}}}\right) \\
& \quad+\alpha \beta \mathcal{N}\left(-X,-\frac{Y}{\sqrt{1+\gamma^{2}}},-\frac{\gamma}{\sqrt{1+\gamma^{2}}}\right)
\end{aligned}
$$

where

$$
\alpha=w \sqrt{T-v}, \beta=a \sqrt{v}, \gamma=\frac{p}{q} \sqrt{\frac{T-v}{v}}
$$

and

$$
X=\frac{y+(T-v) w}{\sqrt{T-v}}, Y=\frac{q k-p y-p w(T-v)+q v a}{q \sqrt{v}} .
$$

Theorem 3 is proved in Section 4.3 . 


\section{Proofs}

\subsection{Proofs of Theorem 1 and Theorem 2}

We prove Theorem 1 only. Theorem 2 can be proved in a similar way with straightforward modifications (see Remark 2).

Given $n \in \mathbb{N}$ consider a discrete time Markov chain $S_{k}^{(n)} \in \mathbb{R}, k \in \mathbb{Z}_{+}$, specified by the following transition probabilities

$$
\begin{aligned}
\mathrm{P}\left(S_{k+1}^{(n)}=x+1 \mid S_{k}^{(n)}=x>0\right) & =\mathrm{P}\left(S_{k+1}^{(n)}=x-1 \mid S_{k}^{(n)}=x>0\right)=\frac{1}{2}, \\
\mathrm{P}\left(S_{k+1}^{(n)}=x+1 \mid S_{k}^{(n)}=x<0\right) & =\mathrm{P}\left(S_{k+1}^{(n)}=x-1 \mid S_{k}^{(n)}=x<0\right)=\frac{1}{2}, \\
\mathrm{P}\left(S_{k+1}^{(n)}=1 \mid S_{k}^{(n)}=0\right) & =p, \\
\mathrm{P}\left(S_{k+1}^{(n)}=-1 \mid S_{k}^{(n)}=0\right) & =q=1-p .
\end{aligned}
$$

Define the following stochastic process

$$
X_{t}^{(n)}=\frac{1}{\sqrt{n}} S_{[n t]}^{(n)}+\frac{n t-[n t]}{\sqrt{n}}\left(S_{1+[n t]}^{(n)}-S_{[n t]}^{(n)}\right), t \geq 0 .
$$

Quantities (91), (8) and (10) for process $X_{t}^{(n)}$ are defined as follows

$$
\tau\left(X^{(n)}\right)=\frac{\tau_{n}}{n}, V\left(X^{(n)}\right)=\frac{V_{n}}{n}, L\left(X^{(n)}\right)=L_{n},
$$

where

$$
\begin{aligned}
\tau_{n} & =\max \left\{k: S_{k}^{(n)}=0\right\}, \\
V_{n} & =\sum_{i=0}^{\tau_{n}} 1_{\left\{S_{i}^{(n)} \geq 0, S_{i+1}^{(n)} \geq 0\right\}}, \\
L_{n} & =\sum_{i=0}^{[T n]} 1_{\left\{S_{i}^{(n)}=0\right\}} .
\end{aligned}
$$

Theorem 11 is implied by Lemmas 2 and 3 below.

Lemma 2 Let $X_{t}^{(n)}$ be the process defined by (35) and let $\tau_{n}, V_{n}$ and $L_{n}$ be quantities defined by (36), (37) and (38). Then

$$
\left(\frac{\tau_{n}}{n}, \frac{V_{n}}{n}, \frac{L_{n}}{\sqrt{n}}, X_{T}^{(n)}\right) \rightarrow\left(\tau, V, L_{T}^{(0)}\left(W^{(p)}\right), W_{T}^{(p)}\right),
$$

in distribution, as $n \rightarrow \infty$.

Proof of Lemma 国. It has been proved in [14] that $X_{t}^{(n)}$ converges in the space of continuous functions, as $n \rightarrow \infty$, to SBM $W_{t}^{(p)}$. This implies the claim of the lemma. 
Lemma 3 Let $X_{t}^{(n)}$ be the process defined by (35) and let $\tau_{n}, V_{n}$ and $L_{n}$ be quantities defined by (36), (37) and (38). Suppose that sequences of numbers $r_{n}, r_{1, n}$ and $k_{n}$ are such that

$$
\frac{2 r_{1, n}}{n} \rightarrow x \in[0, T], \frac{2\left(r_{n}-r_{1, n}\right)}{n} \rightarrow y \in[0, T], \frac{k_{n}}{\sqrt{n}} \rightarrow l \in \mathbb{R}_{+}, \text {as } n \rightarrow \infty,
$$

where $x+y=t \leq T$.

1) If, in addition, $\frac{j_{n}}{\sqrt{n}} \rightarrow z \geq 0$, as $n \rightarrow \infty$, then

$$
\begin{gathered}
\lim _{n \rightarrow \infty} \frac{n^{3}}{8} \mathrm{P}\left(V_{n}=2 r_{1, n}, \tau_{n}=2 r_{n}, L_{n}=k_{n}, X_{1}^{(n)}=j_{n} \mid X_{0}^{(n)}=0\right) \\
=\frac{2 p^{2} q l^{2} z}{[2 \pi x(t-x)(T-t)]^{3 / 2}} e^{-\frac{z^{2}}{2(T-t)}-\frac{l^{2}}{2}\left(\frac{p^{2}}{x}+\frac{q^{2}}{t-x}\right)} .
\end{gathered}
$$

2) If, in addition, $\frac{j_{n}}{\sqrt{n}} \rightarrow z<0$, as $n \rightarrow \infty$, then

$$
\begin{gathered}
\lim _{n \rightarrow \infty} \frac{n^{3}}{8} \mathrm{P}\left(V_{n}=2 r_{1, n}, \tau_{n}=2 r_{n}, L_{n}^{(n)}=k_{n}, X_{1}^{(n)}=j_{n} \mid X_{0}^{(n)}=0\right) \\
=\frac{2 p^{2} q l^{2}|z|}{[2 \pi x(t-x)(T-t)]^{3 / 2}} e^{-\frac{z^{2}}{2(T-t)}-\frac{l^{2}}{2}\left(\frac{p^{2}}{x}+\frac{q^{2}}{t-x}\right)} .
\end{gathered}
$$

Lemma 3 is proved in Section 4.2.

Remark 2 Theorem 2 can be proved by modifying appropriately the proof of Theorem 1. First of all, the transition probabilities of Markov chain $S_{k}^{(n)} \in \mathbb{R}, k \in \mathbb{Z}_{+}$, should be modified as follows

$$
\begin{aligned}
\mathrm{P}\left(S_{k+1}^{(n)}=x+1 \mid S_{k}^{(n)}=x>0\right) & =\frac{1}{2}\left(1+\frac{m_{1}}{\sqrt{n}}\right), \\
\mathrm{P}\left(S_{k+1}^{(n)}=x-1 \mid S_{k}^{(n)}=x>0\right) & =\frac{1}{2}\left(1-\frac{m_{1}}{\sqrt{n}}\right), \\
\mathrm{P}\left(S_{k+1}^{(n)}=x+1 \mid S_{k}^{(n)}=x<0\right) & =\frac{1}{2}\left(1+\frac{m_{2}}{\sqrt{n}}\right), \\
\mathrm{P}\left(S_{k+1}^{(n)}=x-1 \mid S_{k}^{(n)}=x<0\right) & =\frac{1}{2}\left(1-\frac{m_{2}}{\sqrt{n}}\right), \\
\mathrm{P}\left(S_{k+1}^{(n)}=1 \mid S_{k}^{(n)}=0\right) & =p, \\
\mathrm{P}\left(S_{k+1}^{(n)}=-1 \mid S_{k}^{(n)}=0\right) & =q=1-p .
\end{aligned}
$$

Let $X^{(n)}$ be a stochastic process defined by equation (35) as before. Convergence of $X^{(n)}$ to SBM with drift $m$ can be proved by a straightforward modification of the proof in [14] (see also [20]) in the driftless case. Convergence implies an analogue of Lemma 2. It is also rather straightforward to make appropriate changes in both the statement and the proof of Lemma 3 in the case of non-zero drift. We skip the details.

Alternatively, one can combine Theorem 1 and the Girsanov's theorem (see Remark 1) to obtain Theorem 2 , 


\subsection{Proof of Lemma 3}

Recall that $X_{t}^{(n)}$ is the process defined by (351).

Definition 1 Given $n$ consider a discrete trajectory $X_{t_{k}}^{(n)}, k=0,1, \ldots, n$, where we denoted

$$
t_{k}=\frac{k}{n}, k=0,1, \ldots,[T n]
$$

- A part of the trajectory $\left(X_{t_{k}}^{(n)}, X_{t_{k+1}}^{(n)}, \ldots, X_{t_{k+2 d}}^{(n)}\right)$ such that

$$
X_{t_{k}}^{(n)}=0, X_{t_{k+1}}^{(n)}>0, \ldots, X_{t_{k+2 d-1}}^{(n)}>0, X_{t_{k+2 d}}^{(n)}=0,
$$

is called a positive cycle of length $2 d$.

Similar, a part of the trajectory $\left(X_{t_{k}}^{(n)}, X_{t_{k+1}}^{(n)}, \ldots, X_{t_{k+2 d}}^{(n)}\right)$ such that

$$
X_{t_{k}}^{(n)}=0, X_{t_{k+1}}^{(n)}<0, \ldots, X_{t_{k+2 d-1}}^{(n)}<0 X_{t_{k+2 d}}^{(n)}=0
$$

is called a negative cycle of length $2 d$.

- Let $R_{n}$ be the number of positive cycles in a trajectory $X_{t_{k}}^{(n)}, k=0, \ldots,[T n]$.

- Given $r, r_{1}, k, i \in \mathbb{Z}_{+}$, where $r_{1} \leq r$ and $i \leq k$, define the following set of trajectories $X_{t_{k}}^{(n)}, k=0, \ldots,[$ Tn $]$, such that

$$
A_{r, r_{1}, k, i}=\left\{\tau_{n}=2 r, L_{n}=k, R_{n}=i, V_{n}=2 r_{1}\right\} .
$$

Notice that the total number of both positive and negative cycles equals $L_{n}$.

We prove the lemma only if $z \geq 0$ (the case $z<0$ can be considered similar). Given $j \geq 0$ denote

$$
B_{n, r, j}=\left\{X_{t_{2 r+1}}^{(n)}>0, \ldots, X_{t_{[T n]-1}}^{(n)}>0, X_{T}^{(n)}=j\right\} .
$$

It is easy to see that

$$
\begin{aligned}
\mathrm{P}\left(V_{n}\right. & \left.=2 r_{1, n}, \tau_{n}=2 r_{n}, L_{n}=k_{n}, X_{1}^{(n)}=j_{n} \mid X_{0}^{(n)}=0\right) \\
& =\left(\sum_{i=0}^{k_{n}} \mathrm{P}\left(A_{r_{n}, r_{1, n}, k_{n}, i}\right)\right) \mathrm{P}\left(B_{n, r_{n}, j_{n}} \mid X_{t_{2 r_{n}}}^{(n)}=0\right)
\end{aligned}
$$

The statement of the lemma is implied by two following propositions.

Proposition 1 Under assumptions of Lemma 3

$$
\lim _{n \rightarrow \infty} n^{2}\left(\sum_{i=0}^{k_{n}} \mathrm{P}\left(A_{r_{n}, r_{1, n}, k_{n}, i}\right)\right)=\frac{2 p q l^{2}}{\pi(x(t-x))^{3 / 2}} e^{-\frac{l^{2}}{2}\left(\frac{p^{2}}{x}+\frac{q^{2}}{t-x}\right)}=4 h(x, p l) h(t-x, l q) .
$$




$$
\begin{aligned}
\lim _{n \rightarrow \infty} n \mathrm{P}\left(X_{t_{2 r+1}}^{(n)}\right. & \left.>0, \ldots, X_{t_{[T n]-1}^{(n)}}^{(n)}>0, X_{T}^{(n)}=j \mid X_{t_{2 r}}^{(n)}=0\right) \\
& =\sqrt{\frac{2}{\pi}} \frac{2 p z}{(T-t)^{3 / 2}} e^{-\frac{z^{2}}{2(T-t)}}=4 p h(T-t, z) .
\end{aligned}
$$

2) Under assumptions of Part 2) of Lemma [3,

$$
\begin{aligned}
\lim _{n \rightarrow \infty} n \mathrm{P}\left(X_{t_{2 r+1}}^{(n)}\right. & \left.<0, \ldots, X_{t_{[T n]-1}^{(n)}}^{(n)}<0, X_{T}^{(n)}=j \mid X_{t_{2 r}}^{(n)}=0\right) \\
& =\sqrt{\frac{2}{\pi}} \frac{2 q|z|}{(T-t)^{3 / 2}} e^{-\frac{z^{2}}{2(T-t)}}=4 q h(T-t, z) .
\end{aligned}
$$

\subsubsection{Proof of Proposition 1}

We write $r=r_{n}, r_{1}=r_{1, n}$ and $k=k_{n}$ throughout the proof. It is easy to see that probabilities of a positive cycle of length $2 d$ and of a negative cycle of length $2 d$, where $d \geq 1$, are equal to $2 p / 4^{d}$ and $2 q / 4^{d}$ respectively. Therefore a probability of a single path from $A_{r, r_{1}, k, i}$ is equal to

$$
\frac{2^{k} p^{i} q^{k-i}}{2^{2 r}}
$$

Denote by $N_{2 d, i}$ the number of paths of length $2 d$, starting and ending at the origin and formed by $i$ cycles regardless of their signs. It is easy to see that the number of paths of length $2 d$, starting and ending at the origin and formed by $i$ cycles of the same sign is equal to $N_{2 d, i} / 2^{i}$. Therefore, the number of trajectories forming set $A_{r, r_{1}, k, i}$ is equal to

$$
\left(\begin{array}{c}
k \\
i
\end{array}\right) \frac{N_{2 r_{1}, i}}{2^{i}} \frac{N_{2\left(r-r_{1}\right), k-i}}{2^{k-i}} .
$$

Notice that

$$
\frac{N_{2 d, i}}{2^{2 d}}=f_{2 d}^{(i)}
$$

where $f_{2 d}^{(i)}$ is the probability that $i$-th return of SSRW to the origin occurs at time moment $2 d$. Summarising equations (39), (40) and (41) we get the following formula

$$
\mathrm{P}\left(A_{r, r_{1}, k, i}\right)=\left(\begin{array}{c}
k \\
i
\end{array}\right) p^{i} q^{k-i} f_{2 r_{1}}^{(i)} f_{2\left(r-r_{1}\right)}^{(k-i)} .
$$

It is known (Section 7, ch.3, [9]) that

$$
f_{2 d}^{(i)}=\frac{i}{2 d-i} \frac{1}{2^{2 d-i}}\left(\begin{array}{c}
2 d-i \\
d
\end{array}\right) .
$$

If $d$ is large and $i^{2} /(2 d)$ is not very large or close to zero, then the following approximations can be used (equation (7.6) in Section 7, ch.3, [9])

$$
f_{2 d}^{(i)} \approx \sqrt{\frac{2}{\pi}} \frac{i}{(2 d-i)^{3 / 2}} e^{-\frac{i^{2}}{2(2 d-i)}} .
$$


Using this approximation it can be shown that

$$
\left|\sum_{i=0}^{k}\left(\begin{array}{c}
k \\
i
\end{array}\right) p^{i} q^{k-i} f_{2 r_{1}}^{(i)} f_{2\left(r-r_{1}\right)}^{(k-i)}-\frac{2}{\pi} \sum_{i=0}^{k} \frac{\left(\begin{array}{l}
k \\
i
\end{array}\right) p^{i} q^{k-i} i(k-i) e^{-\frac{i^{2}}{2\left(2 r_{1}-i\right)}-\frac{(k-i)^{2}}{2\left(2\left(r-r_{1}\right)-k+i\right)}}}{\left(2 r_{1}-i\right)^{3 / 2}\left(2\left(r-r_{1}\right)-k+i\right)^{3 / 2}}\right| \rightarrow 0
$$

as $n \rightarrow \infty$. Under assumptions of Lemma 3 the second sum in the preceding display can be replaced by the following one

$$
\frac{1}{n^{2}} \frac{2 l^{2}}{\pi(x y)^{3 / 2}} \sum_{i=0}^{k} \frac{\left(\begin{array}{c}
k \\
i
\end{array}\right) p^{i} q^{k-i} i(k-i)}{k^{2}} e^{-\frac{l^{2}}{2}\left(\frac{i^{2}}{k^{2} x}+\frac{1}{y}\left(1-\frac{i}{k}\right)^{2}\right)},
$$

which, in turn, is equal to the expectation $\mathrm{E}\left(F\left(\frac{\xi_{n}}{k}\right)\right)$, where $\xi_{n}$ is a Binomial random variable with parameters $k_{n}$ and $p$, and where function $F$ is defined as follows

$$
F(z)=z(1-z) e^{-\frac{l^{2}}{2}\left(\frac{z^{2}}{x}+\frac{(1-z)^{2}}{y}\right)} .
$$

By Law of Large Numbers

$$
\mathrm{E}\left(F\left(\frac{\xi_{n}}{k}\right)\right) \rightarrow F(p)=p q e^{-\frac{l^{2}}{2}\left(\frac{p^{2}}{x}+\frac{q^{2}}{y}\right)}, \quad \text { as } \quad n \rightarrow \infty
$$

Combining equations (42), (43) and (44) we get that

$$
n^{2} \sum_{i=0}^{k} \mathrm{P}\left(A_{r, r_{1}, k, i}\right)=n^{2} \sum_{i=0}^{k}\left(\begin{array}{c}
k \\
i
\end{array}\right) p^{i} q^{k-i} f_{2 r_{1}}^{(i)} f_{2\left(r-r_{1}\right)}^{(k-i)} \rightarrow \frac{2 p q l^{2}}{\pi(x(t-x))^{3 / 2}} e^{-\frac{l^{2}}{2}\left(\frac{p^{2}}{x}+\frac{q^{2}}{t-x}\right)},
$$

as $n \rightarrow \infty$.

\subsubsection{Proof of Proposition 2}

Proposition 2 is proved in [4], chapter 9, as a part of derivation of the joint distribution of the standard BM, its last visit to the origin and the occupation time. We give the proof here for the sake of completeness and for reader's convenience. For simplicity of notation and without loss of generality, we assume that $[T n]$ is an integer, so that $t_{[T n]}=T$.

It is easy to see that probability of a single trajectory such that

$$
X_{t_{2 r}}^{(n)}=0, X_{t_{2 r+1}}^{(n)}>0, \ldots, X_{t_{[T n]-1}^{(n)}}^{(n)}>0, X_{t_{[T n]}^{(n)}}^{(n)}=X_{T}^{(n)}=j>0,
$$

is equal to $p / 2^{n-2 r-1}$. Therefore,

$$
\begin{aligned}
\mathrm{P}\left(X_{t_{2 r+1}}^{(n)}\right. & \left.>0, \ldots, X_{t_{T n-1}}^{(n)}>0, X_{T}^{(n)}=j \mid X_{t_{2 r}}^{(n)}=0\right) \\
& =2 p \mathrm{P}\left(S_{2 r+1}>0, \ldots, S_{T n-1}>0, S_{T n}=j \mid S_{2 r}=0\right)
\end{aligned}
$$

where $S_{k}$ is the simple symmetric random walk (SSRW). If $T n-2 r$ and $j$ have the same parity, then

$$
\mathrm{P}\left(S_{2 r+1}>0, \ldots, S_{n-1}>0, S_{T n}=j \mid S_{2 r}=0\right)=\frac{j}{T n-2 r} \mathrm{P}\left(S_{T n-2 r}=j \mid S_{0}=0\right) .
$$


It is easy to see that under assumptions of the lemma

$$
\frac{j}{\sqrt{T n-2 r}} \rightarrow \frac{z}{\sqrt{T-t}}
$$

hence, by Local Limit Theorem

$$
\frac{\sqrt{T n-2 r}}{2} \mathrm{P}\left(S_{T n-2 r}=j \mid S_{0}=0\right) \rightarrow \frac{1}{\sqrt{2 \pi}} e^{-\frac{z^{2}}{2(T-t)}} .
$$

We conclude the proof by noticing that

$$
\lim _{n \rightarrow \infty} n \frac{2 j}{(T n-2 r)^{3 / 2}}=\frac{2 z}{(T-t)^{3 / 2}} .
$$

\subsection{Proof of Theorem 3}

Proof of Part 1) of Theorem 3. It is easy to see that if $S_{0}>1$ and $K>1$ then we get the following equation for the option price

$$
\mathrm{C}_{\mathrm{in}}=\int_{k}^{\infty} \int_{0}^{\infty} \int_{\Gamma_{T, 1}}\left(e^{\sigma_{1} x}-e^{\sigma_{1} k}\right) e^{-\left(t_{0}+v+s\right) \lambda_{1}-u \lambda_{2}+\left(x-x_{0}\right) \mu_{1}} h\left(t_{0}, x_{0}\right) \psi_{p, T-t_{0}}(u+v, v, x, l) d t_{0} d x d l d v d s
$$

where $\lambda_{i}=\frac{\sigma_{i}^{2}}{8}, i=1,2, t_{0}$ is the hitting time to zero, $v$ and $u$ are occupation times of the positive half-line and of the negative half-line respectively which are observed between $t_{0}$ and the last visit to the origin (i.e. $\left.t_{0}+v+u\right), s=T-\left(t_{0}+v+u\right), \Gamma_{T, 1}=\left\{\left(t_{0}, v, u, s\right): t_{0}+v+u+s=T\right\}$ and where $\psi_{p, T-t_{0}}$ is given by (11), i.e.

$$
\psi_{p, T-t_{0}}(u+v, v, x, l)=2 p h(v, l p) h(u, l q) h(s, x),
$$

since $x>0$. Using the convolution property of hitting times we get that

$$
\int_{t_{0}+s=t} h\left(t_{0}, x_{0}\right) h(s, x) d t_{0} d s=\int_{0}^{t} h\left(t-s, x_{0}\right) h(s, x) d t d s=h\left(t,\left|x_{0}\right|+|x|\right) .
$$

Notice that $2 p h(v, l p) h(u, l q) h\left(t,\left|x_{0}\right|+|x|\right)=\psi_{p, T}\left(v+u, v,\left|x_{0}\right|+|x|, l\right)$ and rewrite the expression for $\mathrm{C}_{\text {in }}$ as follows

$$
\mathrm{C}_{\mathrm{in}}=\int_{k}^{\infty} \int_{0}^{\infty} \int_{\Gamma_{T, 2}}\left(e^{\sigma_{1} x}-e^{\sigma_{1} k}\right) \psi_{p, T}\left(v+u, v,\left|x_{0}\right|+|x|, l\right) e^{-(t+v) \lambda_{1}-u \lambda_{2}} e^{\mu_{1}\left(x-x_{0}\right)} d l d t d v d x,
$$

where $\Gamma_{T, 2}=\{(t, v, u): t+v+u=T\}$. Denoting

$$
g(u, v)=2 \int_{0}^{\infty} h(v, l p) h(u, l q) d l=\frac{p q}{\sqrt{2 \pi}\left(p^{2} u+q^{2} v\right)^{3 / 2}}
$$


we can rewrite

$$
\mathrm{C}_{\mathrm{in}}=p \int_{k}^{\infty} \int_{\Gamma_{T, 2}}\left(e^{\sigma_{1} x}-e^{\sigma_{1} k}\right) g(u, v) h\left(t,\left|x_{0}\right|+|x|\right) e^{-(t+v) \lambda_{1}-u \lambda_{2}} e^{\mu_{1}\left(x-x_{0}\right)} d t d v d x .
$$

Further, recalling that $\sigma_{1}=-2 \mu_{1}$ we arrive to the following expression for the price

$$
\mathrm{C}_{\text {in }}=p e^{-\frac{\sigma_{1} x_{0}}{2}}\left(F_{\text {call }}\left(\frac{\sigma_{1}}{2}, x_{0}\right)-e^{\sigma_{1} k} F_{\text {call }}\left(-\frac{\sigma_{1}}{2}, x_{0}\right)\right),
$$

where

$$
F_{\text {call }}\left(\alpha, x_{0}\right)=\int_{k}^{\infty} \int_{t+v+u=T} g(u, v) e^{-v \lambda_{1}-u \lambda_{2}} h\left(t,\left|x_{0}\right|+|x|\right) e^{\alpha x} e^{-t \lambda_{1}} d t d v d x
$$

Integrating with respect to variables $u, v$ provided that $u+v=T-t=s$ is fixed we obtain function

$$
\begin{aligned}
F_{1}(s) & =\int_{v+u=s} g(u, v) e^{-v \lambda_{1}-u \lambda_{2}} d v \\
& =\frac{\sigma_{1} \sigma_{2}}{\sigma_{1}-\sigma_{2}}\left[\frac{2}{\sqrt{2 \pi s}}\left(\frac{e^{-\frac{1}{8} s \sigma_{2}^{2}}}{\sigma_{2}}-\frac{e^{-\frac{1}{8} s \sigma_{1}^{2}}}{\sigma_{1}}\right)+\left(\Phi\left(\frac{\sqrt{s} \sigma_{2}}{2}\right)-\Phi\left(\frac{\sqrt{s} \sigma_{1}}{2}\right)\right)\right],
\end{aligned}
$$

defined earlier by equation (33). Integrating out variable $x$ we get

$$
\begin{aligned}
\int_{k}^{\infty} h\left(t,\left|x_{0}\right|+|x|\right) e^{\alpha x} d x & =\frac{1}{\sqrt{2 \pi} \sqrt{t}} e^{k \alpha-\frac{\left(\left|x_{0}\right|+|k|\right)^{2}}{2 t}} \\
& +\alpha e^{\frac{t \alpha^{2}}{2}-\alpha\left|x_{0}\right|}\left(1-\Phi\left(\frac{\left|x_{0}\right|+|k|-t \alpha}{\sqrt{t}}\right)\right) \\
& =F_{2}\left(\alpha, t, x_{0}, 1\right)
\end{aligned}
$$

where function $F_{2}\left(\alpha, t, x_{0}, \theta\right)$ is defined by (34). Finally, we rewrite $F_{\text {call }}$ in terms of $F_{1}$ and $F_{2}$

$$
F_{\text {call }}\left(\alpha, x_{0}\right)=\int_{0}^{T} F_{1}(T-t) F_{2}\left(\alpha, t, x_{0}, 1\right) e^{-\frac{t \sigma_{1}^{2}}{8}} d t
$$

as claimed in (32).

Proof of Part 2) of Theorem [3. If $S_{0}<1$ and $K>1$, then $x_{0}=\phi\left(S_{0}\right)=\frac{\log \left(S_{0}\right)}{\sigma_{2}}<0, k=$ $\phi(K)=\frac{\log (K)}{\sigma_{1}}>0$, and we get, using notation introduced in the proof of Part 1), that $\mathrm{C}_{\mathrm{in}}=\int_{k}^{\infty} \int_{0}^{\infty} \int_{\Gamma_{T, 1}}\left(e^{\sigma_{1} x}-e^{\sigma_{1} k}\right) h\left(t_{0}, x_{0}\right) \psi_{p, T-t_{0}}(v+u, v, x, l) e^{-\lambda_{1}(v+s)-\lambda_{2}\left(t_{0}+u\right)+\mu_{1} x-\mu_{2} x_{0}} d t_{0} d x d l d v d s$ where, as before, $h\left(t_{0}, x_{0}\right) \psi_{p, T-t_{0}}(u+v, v, x, l)=2 p h\left(t_{0}, x_{0}\right) h(v, l p) h(u, l q) h(s, x)$. We use again the convolution property of hitting times as in Part 1) but integrate now products $h(v, l p) h(s, x)$ 
and $h\left(t_{0}, x_{0}\right) h(u, l q)$ given constraints $v+s=$ const and $t_{0}+u=$ const respectively. It leads to the following expression for the price

$$
\begin{aligned}
\mathrm{C}_{\text {in }} & =2 p \int_{0}^{T} \int_{k}^{\infty} \int_{0}^{\infty}\left(e^{\sigma_{1} x}-e^{\sigma_{1} k}\right) h(v, l p+x) h\left(u, l q+\left|x_{0}\right|\right) e^{-\lambda_{1} v-\lambda_{2} u+\mu_{1} x-\mu_{2} x_{0}} d l d x d v \\
& =2 p e^{\frac{\sigma_{2} x_{0}}{2}} \int_{0}^{T} e^{-\frac{\sigma_{1}^{2} v}{8}-\frac{\sigma_{2}^{2} u}{8}} \int_{k}^{\infty} \int_{0}^{\infty}\left(e^{\sigma_{1} x}-e^{\sigma_{1} k}\right) h(v, l p+x) h\left(u, l q+\left|x_{0}\right|\right) e^{-\frac{\sigma_{1} x}{2}} d l d x d v \\
& =2 p e^{\frac{\sigma_{2} x_{0}}{2}} \int_{0}^{T} e^{-\frac{\sigma_{1}^{2} v}{8}-\frac{\sigma_{2}^{2} u}{8}} \int_{k}^{\infty} \int_{0}^{\infty} h(v, l p+x) h\left(u, l q+\left|x_{0}\right|\right) e^{\frac{\sigma_{1} x}{2}} d l d x d v \\
& -2 p e^{\frac{\sigma_{2} x_{0}}{2}+\sigma_{1} k} \int_{0}^{T} e^{-\frac{\sigma_{1}^{2} v}{8}-\frac{\sigma_{2}^{2} u}{8}} \int_{k}^{\infty} \int_{0}^{\infty} h(v, l p+x) h\left(u, l q+\left|x_{0}\right|\right) e^{-\frac{\sigma_{1} x}{2}} d l d x d v
\end{aligned}
$$

where $u=T-v$ and $\mu_{i}=-\sigma_{i} / 2$ and $\lambda_{i}=\sigma_{i}^{2} / 8$. Rewrite

$$
\mathrm{C}_{\text {in }}=2 p e^{\frac{\sigma_{2} x_{0}}{2}} \int_{0}^{T} e^{-\frac{\sigma_{1}^{2} v}{8}-\frac{\sigma_{2}^{2}(T-v)}{8}}\left(\mathrm{I}\left(-\frac{\sigma_{1}}{2},\left|x_{0}\right|, v\right)-e^{\sigma_{1} k} \mathrm{I}\left(\frac{\sigma_{1}}{2},\left|x_{0}\right|, v\right)\right) d v
$$

where

$$
\mathrm{I}(a, y, v)=\int_{k}^{\infty} \int_{0}^{\infty} h(v, l p+x) h(u, l q+y) e^{-a x} d l d x, \quad y \geq 0 .
$$

Notice that

$$
\begin{aligned}
h(v, l p+x) h(u, l q+y) e^{-a x} & =h(v, l p+x, a) e^{\frac{v a^{2}}{2}+a l p} h(u, l q+y) \\
& =e^{\frac{v a^{2}}{2}+\frac{u}{2}\left(\frac{a p}{q}\right)^{2}-a y \frac{p}{q}} h(v, l p+x, a) h\left(u, l q+y,-a p q^{-1}\right)
\end{aligned}
$$

where $h(t, x, b)$ is defined by (31), so we can rewrite

$$
\mathrm{I}(a, y, v)=e^{\frac{v a^{2}}{2}+\frac{T-v}{2}\left(\frac{a p}{q}\right)^{2}-a y \frac{p}{q}} G_{1}\left(a, v, y,-a p q^{-1}\right),
$$

where

$$
G_{1}(a, v, y, w)=\int_{k}^{\infty} \int_{0}^{\infty} h(v, l p+x, a) h(u, l q+y, w) d l d x
$$

It is left to show that $G_{1}(a, y, v, w)$ can be expressed in terms of the standard normal distribution and a bivariate normal distribution. Noticing that

$$
h(v, l p+x, a) h(u, l q+y, w)=\frac{(l p+x)(l q+y)}{2 \pi(u v)^{3 / 2}} e^{-\frac{(l p+x+v a)^{2}}{2 v}-\frac{(l q+y+u w)^{2}}{2 u}}
$$


and changing variables $z_{1}=\frac{l p+x+a v}{\sqrt{v}}$ and $z_{2}=\frac{l q+y+u w}{\sqrt{u}}$ we can rewrite the expression for $G_{1}$ as follows

$$
G_{1}(a, y, v, w)=\int_{D} \frac{e^{-\frac{w_{1}^{2}}{2}-\frac{w_{2}^{2}}{2}}}{2 \pi} \frac{\left(z_{1}-a \sqrt{v}\right)\left(z_{2}-w \sqrt{u}\right)}{q \sqrt{u v}} d z_{1} d z_{2}
$$

where $D=\left\{\left(z_{1}, z_{2}\right) \in \mathbb{R}^{2}: z_{2} \sqrt{u}>y+u w,-z_{2} p \sqrt{u}+q \sqrt{v} w_{2}>q k-p y+q v a-p u w\right\}$. Denote

$$
\alpha=w \sqrt{u}, \beta=a \sqrt{v}, X=\frac{y+u w}{\sqrt{u}}, Y=\frac{q k-p y-p u w+q v a}{q \sqrt{v}}, \gamma=\frac{p}{q} \sqrt{\frac{u}{v}},
$$

and $\Gamma=\left\{\left(z_{1}, z_{2}\right): z_{1}>Y+\gamma z_{2}, z_{2}>X\right\}$. In these notation

$$
G_{1}(a, y, v, w)=\int_{\Gamma} \frac{e^{-\frac{z_{1}^{2}}{2}-\frac{z_{2}^{2}}{2}}}{2 \pi} \frac{\left(z_{1}-\beta\right)\left(z_{2}-\alpha\right)}{q \sqrt{u v}} d z_{1} d z_{2}=\frac{1}{q \sqrt{u v}} \mathrm{~J}(a, y, v, w),
$$

where $\mathrm{J}(a, y, v, w)=\int_{\Gamma} \mathrm{n}\left(z_{1}\right) \mathrm{n}\left(z_{2}\right)\left(z_{1}-\beta\right)\left(z_{2}-\alpha\right) d z_{1} d z_{2}$, and function $\mathrm{n}$ is defined by (28). Notice that

$$
\begin{aligned}
\mathrm{J}(a, y, v, w) & =\int_{\Gamma} z_{1} z_{2} \mathrm{n}\left(z_{1}\right) \mathrm{n}\left(z_{2}\right) d z_{1} d z_{2}-\alpha \int_{\Gamma} z_{1} \mathrm{n}\left(z_{1}\right) \mathrm{n}\left(z_{2}\right) d z_{1} d z_{2} \\
& -\beta \int_{\Gamma} z_{2} \mathrm{n}\left(z_{1}\right) \mathrm{n}\left(z_{2}\right) d z_{1} d z_{2}+\alpha \beta \int_{\Gamma} \mathrm{n}\left(z_{1}\right) \mathrm{n}\left(z_{2}\right) d z_{1} d z_{2} \\
& :=\mathrm{J}_{1}+\mathrm{J}_{2}+\mathrm{J}_{3}+\mathrm{J}_{4} .
\end{aligned}
$$

It can be shown (we skip intermediate computational details) that

$$
\begin{aligned}
& \mathrm{J}_{1}=\frac{\mathrm{n}(\gamma X+Y) \mathrm{n}(X)}{1+\gamma^{2}}-\frac{\gamma Y}{\left(1+\gamma^{2}\right)^{3 / 2}} \mathrm{n}\left(\frac{Y}{\sqrt{1+\gamma^{2}}}\right) \Phi\left(-\frac{\left(1+\gamma^{2}\right) X+\gamma Y}{\sqrt{1+\gamma^{2}}}\right) \\
& \mathrm{J}_{2}=-\frac{\alpha}{\sqrt{1+\gamma^{2}}} \mathrm{n}\left(\frac{Y}{\sqrt{1+\gamma^{2}}}\right) \Phi\left(-\frac{X\left(1+\gamma^{2}\right)+\gamma Y}{\sqrt{1+\gamma^{2}}}\right) \\
& \mathrm{J}_{3}=-\beta \mathrm{n}(X) \Phi(-\gamma X-Y)-\frac{\gamma}{\sqrt{2 \pi\left(1+\gamma^{2}\right)}} \mathrm{n}\left(\frac{Y}{\sqrt{1+\gamma^{2}}}\right) \Phi\left(-\frac{\left(1+\gamma^{2}\right) X+\gamma Y}{\sqrt{1+\gamma^{2}}}\right) \\
& \mathrm{J}_{4}=\alpha \beta \mathcal{N}\left(-X,-\frac{Y}{\sqrt{1+\gamma^{2}}},-\frac{\gamma}{\sqrt{1+\gamma^{2}}}\right)
\end{aligned}
$$

This finishes the proof of the second part of the theorem.

\section{A Black-Scholes approximation}

In this section we derive a surprisingly simple and accurate approximation for the option price which is based on the Black-Scholes (BS) formula. We use the same notation as in Sections 3.2 
and 4.3. Without loss of generality assume that $S_{0}=1\left(x_{0}=0\right)$ and $K>1(k>0)$. In this case $\mathrm{C}=\mathrm{C}_{\text {in }}$ and equation (45) becomes

$$
\mathbf{C}=p \int_{k}^{\infty} \int_{t+u+v=T}\left(e^{\sigma_{1} x}-e^{\sigma_{1} k}\right) g(u, v) h(t, x) e^{-(t+v) \lambda_{1}-u \lambda_{2}} e^{\mu_{1} x} d t d v d x .
$$

The approximation is motivated by the following idea. Since $k>0$ we "should be mostly interested" in those trajectories of $X_{t}$ that spend the "most of their lifetime" in region $X_{t}>0$, where $\sigma=\sigma_{1}$. Therefore, let us first replace function $e^{-(t+v) \lambda_{1}-u \lambda_{2}}$ in (49) by $e^{-\lambda_{1} T}$. Secondly, integrating out variables $v$ and $u=T-t-v$ gives

$$
\int_{0}^{T-t} g(u, v) d v=\int_{0}^{T-t} \frac{p q}{\sqrt{2 \pi}\left(p^{2}(T-t-v)+q^{2} v\right)^{3 / 2}} d v=\frac{2}{\sqrt{2 \pi(T-t)}}=2 \mathrm{p}(0, T-t),
$$

where $\mathrm{p}(y, T-t)$ is the transition density of the standard BM at time $T-t$ so that the result of integration does not depend on $p$ and $q$. Thus, we arrive, after expressing both $\lambda_{1}$ and $\mu_{1}$ in terms of $\sigma_{1}$, to the following approximation for the option price

$$
\begin{aligned}
\mathbf{C} & \approx 2 p \int_{k}^{\infty}\left(e^{\sigma_{1} x}-e^{\sigma_{1} k}\right) e^{-\frac{\sigma_{1}^{2} T}{8}} e^{-\frac{\sigma_{1} x}{2}}\left(\int_{0}^{T} h(t, x) \mathrm{p}(0, T-t) d t\right) d x \\
& =2 p \int_{k}^{\infty}\left(e^{\sigma_{1} x}-e^{\sigma_{1} k}\right) e^{-\frac{\sigma_{1}^{2} T}{8}-\frac{\sigma_{1} x}{2}} \mathrm{p}(x, T) d x \\
& =\frac{2 \sigma_{2}}{\sigma_{1}+\sigma_{2}} \operatorname{BSC}\left(\sigma_{1}\right)
\end{aligned}
$$

where $\operatorname{BSC}\left(\sigma_{1}\right)$ is the $\mathrm{BS}$ price of the option under the log-normal model with volatility $\sigma_{1}$. It is obvious that if we set $\sigma_{1}=\sigma_{2}$ in both sides of the preceding display, then the approximation would become the BS formula for the call option price with volatility $\sigma_{1}$.

Using the same argument we can obtain similar approximation for the put option price. Namely, if $S_{0}=1$, then the price of a put option with strike $K<1$ can be approximated as follows

$$
\text { Put } \approx 2 q \operatorname{BSP}\left(\sigma_{2}\right)=\frac{2 \sigma_{1}}{\sigma_{1}+\sigma_{2}} \operatorname{BSP}\left(\sigma_{2}\right)
$$

where $\operatorname{BSP}\left(\sigma_{2}\right)$ is the BS price of the put option with volatility $\sigma_{2}$. Similar to the case of the call option, the BS approximation provides either an upper bound (if $\sigma_{1}>\sigma_{2}$ ) or a lower bound (if $\sigma_{1}<\sigma_{2}$ ).

A discontinuous (at $K=1$ ) curve shown on the left-hand side of Figure 1 is the implied volatility curve calculated by using the approximation. In this calculation call prices have been used, if $K>1$, and put prices have been used, if $K<1$. A solid curve in the middle of the left side Figure 1 is the implied volatility curve calculated by using the exact formula provided by Theorem 3. It is easy to see that if $\sigma_{1}<\sigma_{2}$ then the BS approximation provides an upper (lower) bound of the price in the case of call (put) options, and, vice versa, if $\sigma_{1}>\sigma_{2}$ 
then the approximation provides a lower (upper) bound for call (put) prices. In this example $\sigma_{1}=0.5<\sigma_{2}=0.9$, therefore the approximate curve is below the exact curve, if $K<1$, and above it, if $K>1$, as expected. The upper dashed curve is the implied volatility curve calculated by using an approximation proposed in [21] for calibration of a LVM with a piecewise volatility (tiled LVM). The latter includes the two-valued LVM as a particular case.

The BS approximation can be improved. Indeed, recall that we must have the put-call parity $\mathrm{C}-$ Put $=K-S_{0}$, which becomes $\mathrm{C}=$ Put, if $K=S_{0}$. The put-call parity does not hold for the approximate prices and we adjust them so that the put-call parity holds at $K=1$. Namely, define the following adjusting factors

$$
A_{\mathrm{cl}}=\frac{p \operatorname{BSC}\left(\sigma_{1}\right)+q \mathrm{BSP}\left(\sigma_{2}\right)}{2 p \operatorname{BSC}\left(\sigma_{1}\right)}, A_{\mathrm{pt}}=\frac{p \operatorname{BSC}\left(\sigma_{1}\right)+q \mathrm{BSP}\left(\sigma_{2}\right)}{2 q \operatorname{BSP}\left(\sigma_{2}\right)}
$$

and redefine the approximate prices as $\widehat{\operatorname{BSC}\left(\sigma_{1}\right)}=A_{\mathrm{cl}} \operatorname{BSC}\left(\sigma_{1}\right)$ and $\widehat{\operatorname{BSP}\left(\sigma_{2}\right)}=A_{\mathrm{pt}} \operatorname{BSP}\left(\sigma_{2}\right)$. By construction, the put-call parity now holds for adjusted prices at $K=1$. This adjustment smooths the approximate implied volatility curve which becomes continuous everywhere. The result of adjustment is shown on the right-hand side of Figure 1, where both the solid line and the upper dashed line are as before, and the new dashed curve is calculated by using adjusted prices. It is quite visible that the adjustment improves the approximation.

Finally, numerical tests showed that accuracy of the approximation improves as the time to expiration becomes smaller, which agrees with intuition.
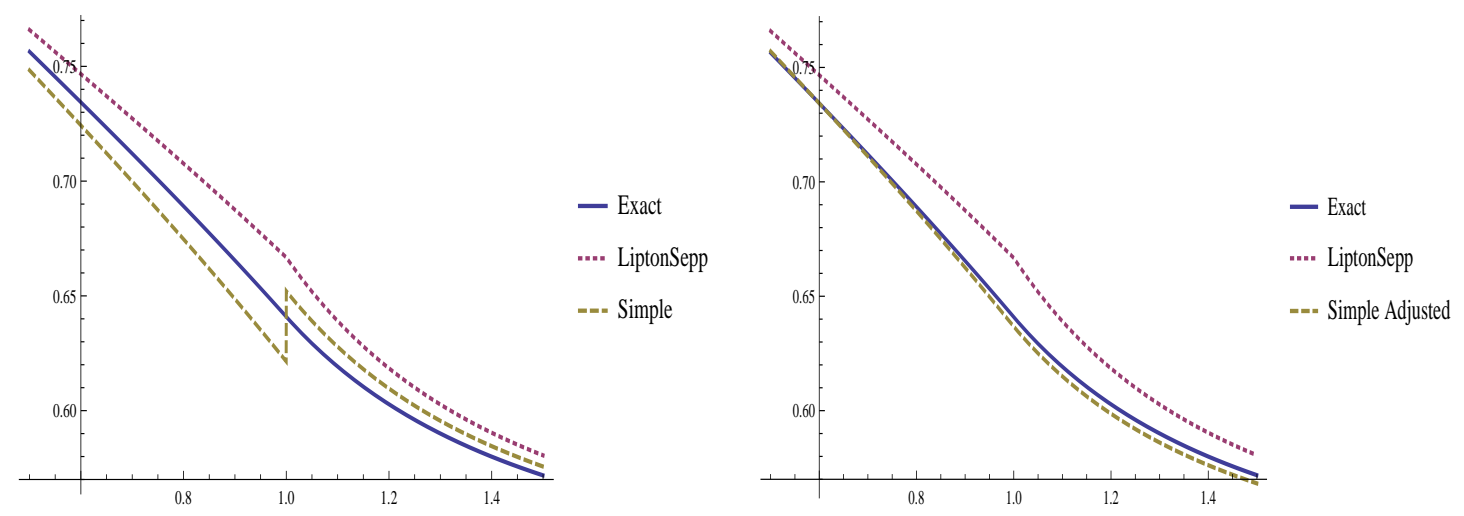

Figure 1: Implied volatility curves, $\sigma_{1}=0.5, \sigma_{2}=0.9, T=2, S_{0}=1$. In both figures: the solid line corresponds to the two-valued LVM and the dashed upper curve corresponds to Lipton-Sepp's approximation. Implied volatility calculated by using BS approximation: without adjustment on the left and with adjustment on the right.

\section{A note on a displaced diffusion model with discontinu- ity}

Our results on the joint distribution of SBM and its functionals can be also applied to derivative pricing in the following displaced model

$$
d S_{t}=\left(\sigma_{1}\left(S_{t}-\alpha_{1}\right) 1_{\left\{S_{t} \geq S^{*}\right\}}+\sigma_{2}\left(S_{t}-\alpha_{2}\right) 1_{\left\{S_{t}<S^{*}\right\}}\right) d W_{t},
$$


where $\sigma_{1} \neq \sigma_{2}, \alpha_{i} \in \mathbb{R}, i=1,2$ and $S^{*}>0$. Model (52) is a particular case of the following model considered in [6]

$$
d S_{t}=\left(\sigma_{1}\left(S_{t}-\alpha_{1}\right)^{\beta_{1}} 1_{\left\{S_{t} \geq S^{*}\right\}}+\sigma_{2}\left(S_{t}-\alpha_{2}\right)^{\beta_{2}} 1_{\left\{S_{t}<S^{*}\right\}}\right) d W_{t}
$$

where, in addition, $\beta_{i} \geq 0, i=1,2$. In [6] they derived certain semi-analytical expressions for the transition density of the underlying process. The technique of [6] is an adaptation of a technique that was used in [11. In turn, the technique of [11] is based on a well known observation (e.g. [10]) that the transition density satisfies a partial differential equation and can be constructed by means of an eigenfunction expansion in the corresponding Sturm-Liouville problem. In general, these eigenfunction expansions for the transition densities are difficult to handle analytically and an approximation is required. It should be noticed that in [6] an analytical expression for the transition density was obtained in a particular case where $\sigma_{1}=\sigma_{2}, \alpha_{1} \neq \alpha_{2}$ so that dependence of the joint density on the occupation time becomes trivial (e.g., see equation (13) or (27), where $m_{1}=m_{2}$ ).

Notice also that if $\sigma_{1}=\sigma_{2}=\sigma, \beta_{1}=\beta_{2}=1$ and $\alpha_{1}=\alpha_{2}=a$ then it is a classical case of a displaced log-normal model. The latter is just $S_{t}=Z_{t}-a$, where $Z_{t}$ is the log-normal process, and it can be written in the local volatility form, namely, $d S_{t}=\sigma\left(1-a / S_{t}\right) S_{t} d W_{t}$. The displaced diffusion is a very useful tool for approximating more complicated stochastic processes in finance. The main reason is that this model is a first-order approximation of any LVM (see Remark 7.2.14 in [1] and other examples therein). A known problem with a displaced model of any sort is that theoretically the underlying process can take negative values (e.g. when $\left.\alpha_{i}>0\right)$. This problem can be dealt with by imposing some constraints. For instance, instead of the classic displaced log-normal model one can consider model (52) with $\alpha_{2}=0$. This means that the volatility is a hyperbolic function above level $S^{*}$ and a constant one below level $S^{*}$ and, hence, is prevented to take large values as the process approaches 0 . It is rather straightforward to apply our results to the displaced log-normal model with such constraints. Let us take, for example, model (52), where $S^{*}=1, \alpha_{1}<1$ and $\alpha_{2}=0$, and consider briefly the case when the process starts at $S_{0}<1$. Given $\sigma_{1}, \sigma_{2}, \alpha_{1}$ and strike $K>1$ define

$$
p=\frac{\sigma_{2}}{\sigma_{2}+\sigma_{1}\left(1-\alpha_{1}\right)}, q=1-p, k=\frac{1}{\sigma_{1}} \log \left(\frac{K-\alpha_{1}}{1-\alpha_{1}}\right), x_{0}=\frac{\log \left(S_{0}\right)}{\sigma_{2}}, b=\frac{q \sigma_{2}-p \sigma_{1}}{2} .
$$

Then the price of a knock-in European call option with strike $K$ and expiration date $T$ is given by the following integral

$$
\mathrm{C}_{\mathrm{in}}=2 p\left(1-\alpha_{1}\right) \int_{k}^{\infty} \int_{0}^{\infty} \int_{\Gamma_{T, 1}}\left(e^{\sigma_{1} x}-e^{\sigma_{1} k}\right) e^{-l \beta-\lambda_{1}(s+v)-x_{0} \mu_{2}-\lambda_{2}\left(t_{0}+u\right)+\mu_{1} x} R\left(u, v, x, l, t_{0}\right) d x d l d v d t_{0}
$$

where $R\left(u, v, x, l, t_{0}\right)=h\left(t_{0}, x_{0}\right) \psi_{p, T-t_{0}}(u+v, v, x, l)$ and we used notation introduced in the proof of Part 1) of Theorem 3. Using the same argument as in the proof of the theorem one can show that computation of the above integral can be reduced to computation of the following integral

$$
\tilde{\mathrm{I}}(b, a, v, y)=\int_{k}^{\infty} \int_{0}^{\infty} e^{-a x-b l} h(v, l p+x) h(u, l q+y) d l d x
$$


In turn, one can express, by modifying appropriately the argument applied to integral (47), the integral in the preceding display in terms of both a univariate and a bivariate normal distribution as follows

$$
\begin{aligned}
\tilde{\mathrm{I}}(b, a, v, y) & =\frac{e^{\frac{\nu^{2} u}{2}+\frac{a^{2} v}{2}+\nu y}}{q \sqrt{u v}}\left(\frac{e^{-\frac{X^{2}+(Y+\gamma X)^{2}}{2}}}{2 \pi\left(1+\gamma^{2}\right)}-\frac{B e^{-\frac{X^{2}}{2}} \Phi(-Y-\gamma X)}{\sqrt{2 \pi}}\right. \\
& +\frac{1}{\sqrt{2 \pi\left(1+\gamma^{2}\right)}}\left(-A+B \gamma-\frac{\gamma Y}{1+\gamma^{2}}\right) e^{-\frac{Y^{2}}{2\left(1+\gamma^{2}\right)}} \Phi\left(-\frac{\left.\left(1+\gamma^{2}\right) X+\gamma Y\right)}{\sqrt{1+\gamma^{2}}}\right) \\
& \left.+A B \mathcal{N}\left(-X,-\frac{Y}{\sqrt{1+\gamma^{2}}},-\frac{\gamma}{\sqrt{1+\gamma^{2}}}\right)\right)
\end{aligned}
$$

where $\nu=-\frac{a p-b}{q}, A=\nu \sqrt{u}, B=a \sqrt{v}, Y=\frac{q(k+a v)-p(\nu u+y)}{q \sqrt{v}}, X=\frac{\nu u+y}{\sqrt{u}}$ and $\gamma=\frac{p}{q} \sqrt{\frac{u}{v}}$.

\section{References}

[1] Andersen L. and Piterbarg V. (2010). Interest Rate Modeling. Atlantic Financial Press.

[2] Appuhamillage, T., Bokil, V., Thomann, E., Waymire, E., Wood, B. (2011): Corrections: Occupation and Local Times for Skew Brownian Motion with Applications to Dispersion Across an Interface. Annals of Applied Probability, 21, N5, pp. 2050-2051.

[3] Appuhamillage, T., Bokil, V., Thomann, E., Waymire, E., Wood, B. (2011). Occupation and local times for skew Brownian motion with applications to dispersion across an interface. Annals of Applied Probability, 21, N1, pp. 183-214.

[4] Billingsley P., (1968). Convergence of Probability Measures. John Wiley\&Sons, Inc.

[5] Decamps, M., De Schepper, A. and Goovaerts, M. (2004). Applications of $\delta$-function perturbation to the pricing of derivative securities. Physica A, 342, pp. 677-692.

[6] Decamps, M., Goovaerts, M., and Schoutens, W. (2006). Self Exciting Threshold Interest Rates Models. International Journal of Theoretical and Applied Finance, 9, N7, pp. 10931122.

[7] Decamps, M., Goovaerts, M., and Schoutens, W. (2006). Asymmetric skew Bessel processes and their applications to finance. Journal of Computational and Applied Mathematics, 186, pp. $130-147$.

[8] Dupire, B. (1994). Pricing with a Smile. Risk, 7(1), pp. 18-20.

[9] Feller, W. (1968). An Introduction to Probability Theory and its Applications. Volume 1, 3rd Edition. John Wiley\&Sons, Inc.

[10] Gikhman, I., and Skorohod, A. (1972). Stochastic differential equations. 
[11] Gorovoi, V. and Linetsky, V. (2004). Blacks model of interest rates as options, eigenfunction expansions and Japanese interest rate. Mathematical finance, 14, N1, pp. 49-79.

[12] Guyon, J. and Henry-Labordere, P. (2011). From spot volatilities to implied volatilities. Risk magazine, $\mathbf{5}$.

[13] Gyongy, I. (1986): Mimicking the One-Dimensional Marginal Distributions of Processes Having an Ito Differential. Probability Theory and Related Fields, 71, pp. 501-516.

[14] Harrison, J. and Shepp, L. (1981). On skew Brownian motion. Annals of Probability, 9, N2, pp. 309-313.

[15] Hull, J. (2009). Options, Futures and Other Derivatives. Pearson/Prentice Hall.

[16] Karatzas, I., and Shreve, (1984). Trivariate density of Brownian motion, its local and occupation times, with application to stochastic control. Annals of Applied Probability, 12, N3, pp. 819-828.

[17] Karatzas, I., and Shreve, S. (1991). Brownian motion and Stochastic Calculus. SpringerVerlag, New York, 2nd Edition.

[18] Ito, K. and McKean, H. (1963). Brownian motions on a half line. Illinois Journal of Mathematics, 7, pp. 181-231.

[19] Le Gall, J.-F. (1985). One-dimensional stochastic differential equations involving the local times of the unknown process. In Stochastic Analysis and Applications. Lecture Notes in Math., 1095. Springer-verlag, pp. 51-82.

[20] Lejay, A. (2006). On the constructions of the skew Brownian motion. Probability Surveys, 3, pp. 413-466.

[21] Lipton, A. and Sepp, A. (2011). Filling the Gaps. Risk, October, pp. 86-91.

[22] Lyulko, Ya. (2012). On the Distribution of Time Spent by a Markov Chain at Different Levels Until Achieving a Fixed State. Theory of Probability and its Applications, 56, N1, pp. 140-149.

[23] Revuz, D. and Yor, M. (1998). Continuous martingales and Brownian motion. SpringerVerlag, 3rd Edition.

[24] Rossello, D. (2012). Arbitrage in Skew Brownian Motion Models. Insurance Mathematics and Economics, 50, N1, pp. 50-56.

[25] Takacs, L. (1995). On the local time of Brownian motion. Annals of Applied Probability, 5, N3, pp. 741-756. 